\title{
Socially Aware Device-to-multi-device User Grouping for Popular Content Distribution
}

\author{
Jianlong Liu', Wen'an Zhou ${ }^{1}$, and Lixia Lin ${ }^{1}$ \\ ${ }^{1}$ School of Computer Science, Beijing University of Post and Telecommunications \\ Beijing, 100876 China \\ [e-mail: lj119880618@bupt.edu.cn, zhouwa@bupt.edu.cn, lixiallx@bupt.edu.cn] \\ ${ }^{*}$ Corresponding author: Wen’an Zhou \\ Received December 17, 2019; revised July 9, 2020; accepted October 31, 2020; \\ published November 30, 2020
}

\begin{abstract}
The distribution of popular videos incurs a large amount of traffic at the base stations (BS) of networks. Device-to-multi-device (D2MD) communication has emerged an efficient radio access technology for offloading BS traffic in recent years. However, traditional studies have focused on synchronous user requests whereas asynchronous user requests are more common. Hence, offloading BS traffic in case of asynchronous user requests while considering their time-varying characteristics and the quality of experience (QoE) of video request users (VRUs) is a pressing problem. This paper uses social stability (SS) and video loading duration (VLD)tolerant property to group VRUs and seed users (SUs) to offload BS traffic. We define the average amount of data transmission (AADT) to measure the network's capacity for offloading BS traffic. Based on this, we formulate a time-varying bipartite graph matching optimization problem. We decouple the problem into two subproblems which can be solved separately in terms of time and space. Then, we propose the socially aware D2MD user selection (SAD2MD-S) algorithm based on finite horizon optimal stopping theory, and propose the SAD2MD user matching (SA-D2MD-M) algorithm to solve the two subproblems. The results of simulations show that our algorithms outperform prevalent algorithms.
\end{abstract}

Keywords: Finite horizon optimal stopping theory, D2MD, Social stability, Video distribution, Offloading BS traffic.

This work was supported by the National Science and Technology Major Project under Grant No. 2018ZX03001019-003. 


\section{Introduction}

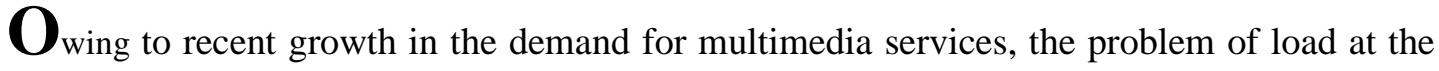
base station (BS) has become significant [1]. A large amount of user-generated contents are transmitted to the network will significantly increased cellular networks load [2]. According to a report by Cisco, mobile data traffic in cellular networks in 2020 is expected to be nine times that in 2014 [3]. To deal with this traffic, a number of radio access technologies have been developed, and device-to-device (D2D) communication is one of them. It decouples the data transmission process from the BS that helps offloading BS traffic to a certain degree. Moreover, when many video request users (VRUs) request the same popular video from the BS at the same time, the requests follow Zipf's distribution [4]. This means that most VRUs have requests in common for content. Therefore, device-to-multi-device (D2MD) communication can significantly offload BS traffic.

The key technologies in D2MD include seed user (SU) selection, user grouping and resource management [5]. Related research has usually treated them together but they have different focuses. Because VRUs can obtain popular videos from SUs, a greater number and higher quality of SUs can provide VRUs with more and better access options for offloading BS traffic, such as by minimizing interference or maximizing energy efficiency [6][7]. To incentivize users to become SUs, energy saving is the most important factor [8]-[10]. Then, VRUs are grouped with them. The quality of service (QoS) of D2MD is affected by the VRU with the worst signal-to-noise ratio (SNR) in the D2MD group incurred by channel quality [11]. Some methods have been proposed to address these problems [12]-[14]. Furthermore, some user grouping schemes have been proposed that consider the energy factor of the VRU [15][16], or combine it with channel quality [17][18]. Based on user groups, some researchers have focused on reusing resources of cellular users in D2MD groups for offloading BS traffic. Maximizing throughput is the most direct and effective method from the perspective of SU. The single data rate [19]-[23] and multiple data rate demands [24][25] of VRUs have been considered. Some methods have been proposed to minimize energy consumption [26] and guarantee fairness from VRUs [27] for offloading BS traffic.

All the above studies focused on synchronous user requests. However, asynchronous user requests are more common. Therefore, a D2MD scheme for this scenario is needed to offload BS traffic while considering the time-varying characteristics and quality of experience (QoE) of VRUs. On the one hand, for different scenarios, different social attributes showed by socially aware (SA) information are used, specially, social stability (SS) is one of those attributes that indicates the stability of communication, i.e., the video request user has available transmission time within the physical distance constraint between idle seed user in the D2D [28]. On the other hand, video loading duration (VLD), a key parameter of QoE indicates even if the SU do not starts a D2MD transmission process immediately after receiving requests from VRUs, and the QoE of VRUs can be guaranteed also [29][30]. In short, based on these two properties, the BS can use a period for optimal D2MD user grouping. Hence, the above problem can be solved. Selecting and matching the optimal VRUs based on this property for a transmission process is an outstanding challenge. The main contributions of this paper are as follows:

(1) We model a BS-assisted SA-D2MD user grouping scenario for asynchronous user requests for the distribution of popular videos as a time-varying bipartite graph matching problem. The physical and SA information between SUs and VRUs, and the VLD-tolerant property of the VRUs are considered to group them with SUs to offload the BS traffic. We 
define the average amount of data transmission (AADT) during a SA-D2MD transmission process to measure the capacity for offloading the BS traffic.

(2) We decouple the problem into two subproblems which can be solved separately from time and space.

- In terms of time, we model the subproblem of optimal multicast time selection. To maximize the AADT with the QoE constraint, finite horizon optimal stopping theory is used to determine the AADT threshold at each time slot. Then, SA-D2MD user selection algorithm (SA-D2MD-S) is proposed.

- In terms of space, we model the subproblem of optimal VRU clustering. Because the VRUs have multiple choices for SUs, the matching from space is not unique. Hence, we divide the subproblem into three problems and develop a heuristic SA-D2MD user matching (SA-D2MD-M) algorithm to solve it.

(3) The results of simulations show that the SA-D2MD-S and SA-D2MD-M\&S algorithms have advantages in terms of AADT with different parameters compared with other algorithms. The remainder of this paper is organized as follows. In Section 2, we provide a review of related work. In Section 3, the system model is described. We then formulate the BS-assisted SA-D2MD user grouping problem in two subproblems in Section 4, solve them in Section 5 by using finite horizon optimal stopping theory and a heuristic search algorithm. In Section 6, we present the results of simulation experiments to test the performance of the SA-D2MD-S and SA-D2MD-M\&S algorithms. Finally, the conclusions of this paper are given in Section 7.

\section{Related Work}

Multimedia data have the characteristics of human centrism [31]. SA information can be used to improve the performance of D2MD for video distribution [28][32]. We review representative studies here. Owing to different time-varying characteristics of user requests, the relevant work can be divided into synchronous user request-based methods and asynchronous user request-based methods.

Synchronous user request-based methods: The assumption of a synchronous user request indicates that the network can be optimized from the spatial perspective based on the given time context-aware information only, and the future status of the network is not considered. A strong social tie represents strong altruism between SUs and VRUs. Not only does it mean longer available transmission time and higher contact probability, it also implies stronger selection preference in the content pull service. Hence, Li et al. [33], Gong, et al. [34] and Zhao et al. [35] used this property to optimize D2MD. Further, social tie also means demand similarity. SU selection, user grouping, and power and channel allocation in content push service, such as in work by Tulu et al. [36], Huang et al. [37], Feng et al. [38], and Wu et al. [39]. Moreover, Bai et al. [40] proposed a hybrid pull/push scheme by D2MD based on an social tie threshold. A weak social tie represents strong selfishness between SUs and VRUs. This means that users without social tie are less likely to communicate with one another. It is necessary to design corresponding incentive mechanism to solve this problem. Hence, Huang et al. [41] designed a proper SU selection and user grouping scheme for the maximum weight. Using more context-aware information, such as interest similarity and geographical location, Cao et al. [42], Zhao et al. [43], and Wang et al. [44] designed user grouping incentive schemes for D2MD. In general, synchronous user request-based methods have the advantages of low time complexity and easy deployment. However, it is difficult to make corresponding adjustments according to the spatiotemporal variation in network state caused by the time- 
varying characteristics of user request, such as changes in channel quality, energy, user numbers, and SA information.

Asynchronous user request-based methods: The assumption of an asynchronous user request indicates that network optimization is on dynamic physical and SA information collected from the perspectives of time and space. Based on changes in channel quality and battery capacity caused by asynchronous user requests, the SU can be updated in time for asynchronous and multiple transmissions, such in Araniti et al. [45] and Jiang et al. [46]. Based on change in user numbers, Wang et al. [47] proposed physical-social distance in the Chinese Restaurant Process (PSD-CRP) for user grouping based on the classic CRP [48]. The selection preference of the VRUs was introduced to improve throughput. Similar work has been reported by Ren et al. [49] for user grouping by PSD-CRP. It is based on social tie, battery capacity, and channel quality. Considering the problem of downloading multiple contents in D2MD, Zhang et al. [50] proposed a user group method to improve throughput based on the Indian Buffet Process (IBP). Based on time-varying social tie, Zhang et al. [51] proposed an social tie increment maximization problem. Selfishness has also been considered in incentive mechanisms. Zhao et al. [52] considered the spatiotemporal variation in network states to incent asynchronous arriving users as SUs. Relevant theories in game theory have been used, such as in Wu et al.'s [53] pricing-based method, Chen et al.'s [54] contract-based method, and Zhu et al.'s [55] payment-based method. With spatiotemporal variation in the physical and SA information, Semiari et al. [56] proposed a self-organizing algorithm to match SUs and spectral resources.

Summary: Although previous schemes have considered asynchronous user request and spatiotemporal variation in physical and SA information to optimize the network for improve throughput and spectral efficiency etc., but they only consider SU updates [45][46] and incentive [52]-[56], and content caching [47]-[51]. In other words, they did not consider one D2MD process time and guaranteeing the QoE of VRUs, so they did not need to decide when to begin a D2MD process. Actually, D2MD is possible even if VRU arrival at different times. Thus, in this paper, we design an optimal stop waiting rule, the ISU actively begins a SAD2MD process over a given period based on the QoE demand of VRUs.

\section{System Model}

In this paper, we consider a scenario involving the distribution of a popular video within the BS cell, as shown in Fig. 1. A large video is cached in the BS and SUs. A large number of VRUs initiate requests for it. To guarantee the QoE of the VRUs, we assume that its VLD threshold is $\boldsymbol{T}$.

The BS is the central control unit of this system, and many users are covered in its range. The BS obtains the physical and SA information for all of them. The physical information is obtained from measurement reports, and SA information is obtained and calculated from online social networks, such as QQ and WeChat, and/or offline social networks such as user contact history. We assume the long-term VRU arriving and SS between VRU and SU are stable. The BS divides users that it covers into two classes. 


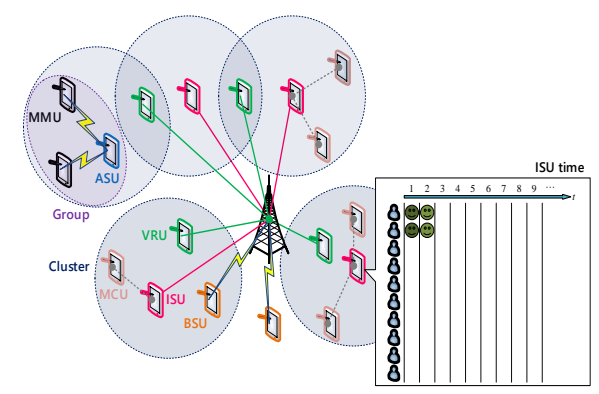

Fig. 1. System scenario of SA-D2MD at given system time

Seed user (SU): Users who have cached the popular video are called SUs. They are preselected by the BS.

Video request users (VRU): Users who asynchronously initiate requests for the popular video, and are VLD tolerant. Each VRU has a different value of SS with different SU.

The SUs are divided into two classes based on their states, as follows:

Active seed users (ASU): SUs who have started multicast.

Idle seed users (ISU): SUs who have not yet started multicast.

An ASU becomes an ISU when a D2MD transmission process is complete. An ISU becomes an ASU after identifying an optimal VRU set.

The VRUs are divided into three classes based on their relationships with the BS or SUs, as follows:

BS direct transmission user (BSU): These VRUs are matched with the BS.

Multicast member user (MMU): These VRUs are matched with ASUs. They receive videos from the ASUs.

Multicast candidate user (MCU): These VRUs are matched with ISUs. They wait for the ISUs to identify the optimal MMU set.

When the ISUs become ASUs, the MCUs turn into MMUs. When the MCUs are leave the ISUs or ASUs, they turn into BSUs.

Based on the SUs, the BS divides all users as clusters, where each cluster contains only one SU and many VRUs. Each group contains an ISU/ASU and some MCUs/MMUs.

Common symbols and definitions in the paper are shown in Table 1, unless otherwise specified.

Table 1. Symbols and definitions

\begin{tabular}{|l|l||l|l|}
\hline \multicolumn{1}{|c|}{ Symbols } & \multicolumn{1}{|c|}{ Definitions } & \multicolumn{1}{c|}{ Symbols } & \multicolumn{1}{c|}{ Definitions } \\
\hline \hline$W$ & Bandwidth. & $P$ & Transmission power. \\
\hline$N_{0}$ & Background noise. & $\alpha$ & Path loss exponent. \\
\hline$D_{p q}$ & $\begin{array}{l}\text { The normalized physical distance } \\
\text { between ISU } p \text { and VRU } q \text {, and } \\
D_{p q} \sim U(0,1) .\end{array}$ & $T$ & $\begin{array}{l}\text { The waiting threshold of the user. } \\
\text { It is discrete, the length of a time } \\
\text { slot is } \tau .\end{array}$ \\
\hline$I$ & $\begin{array}{l}\text { System time. When at least one } \\
\text { ISU is selected by the BS, the } \\
\text { system time begins timekeeping. } \\
\text { It is used to describe the state of } \\
\text { the entire system in terms of time. }\end{array}$ & $i$ & $\begin{array}{l}\text { ISU time. When at least one VRU } \\
\text { is matched with the ISU, the ISU } \\
\text { time begins timekeeping. }\end{array}$ \\
\hline
\end{tabular}




\begin{tabular}{|c|c|c|c|}
\hline$\overline{\mathcal{I}}$ & The ISU set. & $\mathcal{A}$ & The ASU set. \\
\hline $\mathcal{V}$ & The VRU set. & $\mathcal{N}$ & $\begin{array}{l}\text { The new come VRU set, and the } \\
\text { new come VRU set of ISU } p \text { is } \\
\text { denoted by } \mathcal{N}_{p} \text {, and assuming } \\
\mathcal{N}_{p} \sim \pi(\lambda) \text { [57]. }\end{array}$ \\
\hline $\mathcal{C}$ & $\begin{array}{l}\text { The MCU set, and the MCU set } \\
\text { of ISU } p \text { is denoted by } \mathcal{C}_{p} \text {, the } \\
\text { element } s \text { of it is denoted by } \mathcal{C}_{p s} \text {. }\end{array}$ & $\boldsymbol{C}^{(I)}$ & The $\bullet$ set at system time $I$. \\
\hline $\mathbb{E}$ & The expectation. & $\mathrm{ID}$ & The variance. \\
\hline
\end{tabular}

The multicast data rate of D2MD depends on the user with the worst SNR in the MCU set. As follow as [57], we denote

$$
\Upsilon_{p s}=\frac{P}{N_{0} D_{p s}^{\alpha}}[57]
$$

And we denote $\gamma_{p}=\min _{s \in \mathcal{C}_{p}} \Upsilon_{p s}$. Hence, the multicast data rate of ISU $p$ is

$$
R_{p}=W \log _{2}\left(1+\gamma_{p}\right)
$$

For SS, it means a period when ISU $p$ and VRU $q$ are near each other, i.e., the VRU has available transmission time within the physical distance constraint between ISU's in the D2D, which is a common assumption $G_{p q} \sim \operatorname{Gamma}(\delta, \varepsilon)$ [57]. We know that

$$
\begin{aligned}
& \mathbb{E} G=\delta / \varepsilon \\
& \mathbb{D} G=\delta / \varepsilon^{2}
\end{aligned}
$$

Further, we define the remaining available transmission time and VLD for MCU $s$ at ISU $p$ are $A_{p s}$ and $W_{p s}$. Clearly,

$$
A_{p s}=G_{p s}-W_{p s}
$$

Fig. 2 shows that some MCUs are waiting for the ISU to multicast the video to them. In Fig. 2, the green faces represent MCUs that are waiting; some of them are new. Moreover, a deeper color represents a higher SNR. When an MCU leaves, it is represented by a white face in Fig. 2.

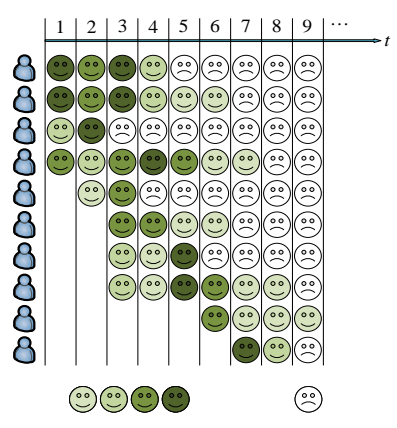

Fig. 2. SA-D2MD user selection

If ISU $p$ begins to multicast, the amount of data transmission of it is denoted by 


$$
L_{p}=\sum_{s \in \mathcal{C}_{p}} \sum_{j=1}^{A_{p s}} R_{p}^{(j)}
$$

Definition 1: We call

$$
H_{p}^{(i)}=\frac{L_{p}^{(i)}}{i+\max _{s \in \mathcal{C}_{p}} A_{p s}}
$$

the average amount of data transmission (AADT) of ISU $p$ when the first VRU arrives and the last MMU leaves.

The AADT of the entire system can be calculated further. However, as the AADT of the entire system is a fixed value at the given system time, to describe the change of amount of data transmission directly, we have

Definition 2: We call

$$
\Delta L^{(I)}=\sum_{p \in \mathcal{V}^{(t)}} L_{p}^{(I)}-\sum_{p \in \mathcal{V}^{(t-1)}} L_{p}^{(I-1)}
$$

the increment of the total amount of data transmission of all ISUs at system time $I$.

\section{Problem Description}

The goal of this paper is to use physical and SS information of ISUs and VRUs to maximize the AADT of D2MD while guarantee the QoE of the VRUs. Fig. 3 shows that each ISU has been matched with some MCUs, it has differences in terms of SNR and SS between MCUs.

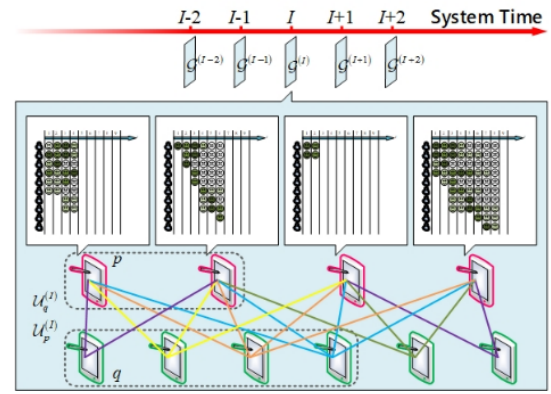

Fig. 3. SA-D2MD user matching

We describe the problem as time-varying bipartite graph matching. A time-varying bipartite graph $\mathcal{G}^{(I)}=\left\langle\mathcal{I}^{(I)}, \mathcal{V}^{(I)}, \mathcal{E}^{(I)}\right\rangle$ is constructed as follows: If ISU $p$ and VRU $q$ meet the physical distance constraint, and there is an edge $e_{p q} \in \mathcal{E}^{(I)}$ in $\mathcal{G}^{(I)}$.

Definition 3: In this paper, we call $\mathcal{U}_{p}=\left\{q \mid q \in \mathcal{V}, e_{p q} \in \mathcal{E}\right\}$ the associated VRU set of ISU $p$. Similarly, we call $\mathcal{U}_{q}$ the associated ISU set of VRU $q$.

Definition 4: This paper denotes by $\mathbf{X}_{p q}=\left(x_{p q}\right)_{|\mathcal{I}| \times|\mathcal{V}|}$ the matching indicator, where

$$
x_{p q}=\left\{\begin{array}{l}
1, e_{p q} \text { is selected } \\
0, e_{p q} \text { is not selected }
\end{array}\right.
$$


where $q=1,2, \cdots,|\mathcal{V}|$ indicates

$$
\sum_{p \in \mathcal{I}} x_{p q}=1
$$

We call $\mathbf{x}_{p q}$ the matching scheme.

Hence, the optimisation problem of time-varying bipartite graph matching is formulated as

$$
P: \mathbf{X}_{p q}^{*}=\underset{i \leqslant T}{\operatorname{argmax}} H_{p}^{(i)}
$$

However, because the problem is highly coupled in term of time and space, it is difficult to find the optimal solution directly. Thus, in order to solve this problem, we simplify the timevarying bipartite graph matching problem. We decouple it from time and space into two subproblems, and solve them independently to find near-optimal solution.

Specifically, when VRU requests the popular video, in terms of space, the BS based on SNR and SS between VRUs and SUs to divide VRUs into different clusters for higher AADT and lower VLD. Once a VRU is matched to a SU, it will not changes utill the VRU away from the SU. Then, in terms of time, after VRUs are matched to SUs, in order to maximize AADT and minimize VLD, each SU need to decide what time it begin a D2MD process. For this, each SU makes a series of AADT threshold based on finite horizon optimal stopping theory.

\section{Subproblem B: Optimal VRU clustering problem}

The BS makes the decision to divide VRUs to maximize the AADT of the system in term of space. In other words, the problem is to find $\mathbf{X}_{p q}$ to maximize the AADT of clusters while guaranteeing QoE by D2MD. The subproblem is described as follows:

$$
P B: \mathbf{X}_{p q}^{*}=\operatorname{argmax} \Delta L^{(I)}
$$

s.t.

$$
\begin{aligned}
& C 1: \sum_{p \in \mathcal{I}} x_{p q}=1 \\
& C 2: x_{p q} \in\{1,0\} \\
& C 3: q=1,2, \cdots,|\mathcal{V}|
\end{aligned}
$$

\section{Subproblem A: Optimal multicast time selection}

Each ISU makes the decision to multicast independently to maximize the AADT only in term of time. In other words, determining the value of $i$ to maximize the AADT of an ISU while guaranteeing QoE is the problem. Therefore, each ISU decides whether to begin a D2MD transmission process by comparing the AADT estimate value with AADT threshold at present time slot. This subproblem is described as follows:

$$
P A: \max _{i \leqslant T} H_{p}^{(i)}
$$

For the convenience of the solution, the optimization in terms of time is first solved, i.e., subproblem A; And then, the optimization in terms of space is solved, i.e., subproblem B. 


\section{Problem Solution}

\subsection{Solution of Subproblem A}

\subsubsection{Finite Horizon Optimal Stopping Theory}

Finite horizon optimal stopping theory is used to maximize the expected reward based on a reward function, where the upper bound on the number of stages at which one may stop is known. For example, in the classic house-selling problem, purchasers want to buy a house of which you are the seller. You do not know their bids before they come, but you may assume that the purchasers are independent and identically distributed. You must decide whether to accept an offer by a purchaser or wait for a better one.

Without loss of generality, for finite horizon optimal stopping theory with horizon $T$, reward function $H$ is needed for the observer to evaluate the reward function at the $i$ th time slot. The reward at the $i$ th time slot is $H^{(i)}$. Denote by $h^{(i)}$ a random variable to represent $\tilde{H}^{(i)}$ from a known distribution. Based on the random series $h^{(0)}, h^{(1)}, \cdots, h^{(i)}, \cdots$, the observer decides whether to stop observing.

The goal of the observer is to find $i=\max _{i=0,1, \cdots, T} \mathbb{E} H^{(i)}$. Hence, the observer needs a threshold series $\Theta^{(0)}, \Theta^{(1)}, \cdots, \Theta^{(i)}$, where $\Theta^{(i)}=\mathbb{E} H^{(i+1)}$. Once $h^{(i)} \geqslant \Theta^{(i)}$, the optimal reward $H^{(i)}$ is obtaining by the observer [58]. The optimal stopping rule for it has the following form:

$$
i^{*}=\min \left\{i \geqslant 1: H^{(i)} \geqslant \mathbb{E} H^{(i+1)}\right\}[59]
$$

\subsubsection{Problem Solving Based on Finite Horizon Optimal Stopping Theory}

$P A$ is a finite horizon optimal stopping problem. There always exists an optimal stopping rule for it. Based on finite horizon optimal stopping theory, the reward threshold is pursued. We denote the reward threshold by $\Theta_{p}^{(i)}$, and $\Theta_{p}^{(i)}=\mathbb{E} H_{p}^{(i+1)}$. Hence, $\mathbb{E} H_{p}^{(i+1)}$ should be calculated as follows.

From Definition $1, H_{p}^{(i)}$ is the mean value of $L_{p}^{(i)}$ in the multicast transmission process. Therefore, we tactfully convert the calculation of the multicast transmission process into that of each time slot. For $i$, this is an obvious observation value. It does not need to be calculated. However, for $\max A_{p s}$, the calculation of its expectation is complicated. For the sake of simplicity, the AADT is represented as Eq. (14):

$$
h_{p}^{(i)}=\frac{L_{p}^{(i)}}{i+\varsigma}
$$

$\varsigma(\varsigma \geqslant 0)$ is called the estimation factor.

We redenote the reward threshold by $\theta_{p}^{(i)}$, and $\theta_{p}^{(i)}=\mathbb{E} h_{p}^{(i+1)}, i=0,1, \cdots, T$. For $\mathbb{E} h_{p}^{(i+1)}$, we have

$$
\mathbb{E} h_{p}^{(i+1)}=\frac{\mathbb{E}\left|\mathcal{C}_{p}^{(i+1)}\right| \mathbb{E} R_{p}^{(i+1)} \mathbb{E} A_{p s}}{i+\varsigma}
$$

Remark 1: Based on the theorem in [60], $\mathbb{E}\left|\mathcal{C}_{p}^{(i+1)}\right|$ is calculated by Eq. (16). 


$$
\mathbb{E}\left|\mathcal{C}_{p}^{(i+1)}\right|=\lambda \int_{i}^{i+1}[1-\operatorname{Gamma}(\delta, \varepsilon)] \mathrm{d} g_{p s}
$$

Remark 2: $\mathbb{E} A_{p s}$ is calculated by Eq. (17).

$$
\mathbb{E} A_{p s}=\frac{\varepsilon^{\delta}}{\Gamma(\delta)} \int_{0}^{+\infty} a_{p s}\left[\int_{0}^{+\infty}\left(a_{p s}+w_{p s}\right)^{\delta-2} e^{-\varepsilon\left(a_{p s}+w_{p s}\right)} \mathrm{d} w_{p s}\right] \mathrm{d} a_{p s}
$$

Then, the calculation of $\theta_{p}^{(i)}$ is converted to calculate $\mathbb{E} R_{p}^{(i+1)}$. We denote the threshold of $\mathbb{E} R_{p}^{(i+1)}$ by $\vartheta_{p}^{(i)}$, i.e., $\vartheta_{p}^{(i)}=\mathbb{E} R_{p}^{(i+1)}$.

Lemma 1: Denoting $f_{R_{p}^{(i+1)}}$ as the probability density function (PDF) of $R_{p}^{(i+1)}$, we have

$$
\begin{aligned}
f_{R_{p}^{(i+1)}}\left(r_{p}^{(i+1)}\right)=\frac{2^{\frac{r_{p}^{(i+1)}}{W}}\left|\mathcal{C}_{p}^{(i+1)}\right| C \ln 2}{W}\left(2^{\frac{r_{p}^{(i+1)}}{W}}-1\right)^{-\frac{1}{\alpha}-1} \cdot \\
\left.\qquad 1-C \alpha\left[\left(\frac{P}{N_{0}}\right)^{-\frac{1}{\alpha}}-\left(2^{\frac{r_{p}^{(i+1)}}{W}}-1\right)^{-\frac{1}{\alpha}}\right]\right\}^{\left|\mathcal{C}_{p}^{(i+1)}\right|-1}
\end{aligned}
$$

It follows that $C \triangleq \frac{1}{B\left(\frac{1}{\alpha}, 1\right)} \frac{P^{\frac{1}{\alpha}}}{N_{0}^{\frac{1}{\alpha}}}$.

Proof: The proof using properties of Beta function. Due to space constraints, we omitted the proof process.

Theorem 1: $\vartheta_{p}^{(i)}$ is calculated as Eq. (19).

$$
\vartheta_{p}^{(i)}=\left\{\begin{array}{l}
-\infty, i=T \\
\int_{M i n R_{p}^{(i)}}^{M a x R_{p}^{(i)}} r_{p}^{(i)} f_{R_{p}^{(i)}}\left(r_{p}^{(i)}\right) d r_{p}^{(i)}, i=T-1 \\
\int_{\vartheta_{p}^{(i)}}^{\operatorname{Max} R_{p}^{(i)}} r_{p}^{(i)} f_{R_{p}^{(i)}}\left(r_{p}^{(i)}\right) d r_{p}^{(i)}+\vartheta_{p}^{(i)} \int_{M i n R_{p}^{(i)}}^{\vartheta_{p}^{(i)}} f_{R_{p}^{(i)}}\left(r_{p}^{(i)}\right) d r_{p}^{(i)}, i=0,1, \cdots, T-2
\end{array}\right.
$$

Proof: Based on Lemma 1, and the backward induction method of finite horizon optimal stopping theory, Eq. (19) can be proof.

\subsubsection{SA-D2MD-S Algorithm}

Based on the theorem 1, we proposed the SA-D2MD user selection algorithm (SA-D2MD-S) to get the solution:

Algorithm 1. SA-D2MD user Selection algorithm (SA-D2MD-S).

Initialization: System parameter.

// Part 1: Offline Part

Step 1: Set $\vartheta_{p}^{(i)} \leftarrow-\infty$

Step 2: For each $i \in[T-1,1]$

Calculate $\vartheta_{p}^{(i)}$ by Eq. (19)

Step 3: Calculate $\mathbb{E}\left|\mathcal{C}_{p}^{(i+1)}\right|$ and $\mathbb{E} A_{p s}$ by Eq. (16) and Eq. (17) respectively 


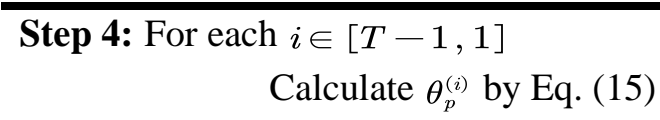

Calculate $\theta_{p}^{(i)}$ by Eq. (15)

// Part 2: Online Part

Step 5: For each $i \in[1, T]$

Calculate $h_{p}^{(i)}$ by Eq. (14)

If $h_{p}^{(i)} \geqslant \theta_{p}^{(i)}$

Find $i^{*}$

Break

Remark 3: The SA-D2MD-S is divided into offline and online parts. The basic operation of the offline part is to calculate expectation, and its time complexity can be denoted by $O(2(T-1)+2)=O(n)$. The basic operation of the online part is to calculate the AADT, and its time complexity can be denoted by $O(T)=O(n)$. Therefore, the overall time complexity of the SA-D2MD-S can be denoted by $O(n)$.

\subsection{Solution of Subproblem B: Heuristic Search Algorithm}

\subsubsection{Problem Analysis}

For $P B, L^{(I-1)}$ is assumed to be constant in the given time slot in this paper. Hence, $L^{(I)}$ is all we need to consider. For Eq (8), $\left|\mathcal{A}^{(I)}\right|, A_{p s}$, and $R_{p}^{(I)}$ are not only functions of $\mathbf{X}_{p q}^{(I)}$, i.e., their values depend on the matching scheme at each time slot, but are also coupled. For instance, $R_{p}^{(I)}$ relies on the matching results, and $\left|\mathcal{A}^{(I)}\right|$ relies on $A_{p s}$ and $R_{p}^{(I)}$. Hence, $L^{(I)}$ is a compound function of $\left|\mathcal{A}^{(I)}\right|, A_{p s}$, and $R_{p}^{(I)}$. This indicates that $P B$ is a compound implicit function 0-1 integer programming problem. Because the problem is difficult to solve, a heuristic SA-D2MD user matching (SA-D2MD-M) algorithm is given as follows:

First, $P B$ is converted into three problems heuristically, i.e., the inner functions $\left|\mathcal{A}^{(I)}\right|, A_{p s}$, and $R_{p}^{(I)}$ are optimized from their independent perspectives. Second, the three problems are analyzed separately, and the rationality of the heuristic conditions of each is given. Third, we propose the heuristic SA-D2MD-M algorithm to weigh the solutions of the three problems above.

\subsubsection{Problem Solution}

(1) Problem conversion

If more ISUs reach the reward threshold, this indicates that more ISUs can become ASUs after matching, and then more $L^{(I)}$ are obtained. If VRUs with higher SS are matched with ISUs, it indicates that more $A_{p s}$ can be used after matching. Then, more $L^{(I)}$ can also be obtained. If the VRUs are matched with ISUs for higher values of $R_{p}^{(I)}$, this indicates that a lower loss of $L^{(I)}$ is obtained. Based on the above heuristic conditions, $P B$ is transferred into three single-objective optimization problems: 


$$
\left\{\begin{array}{l}
P B 1: \mathbf{X}_{p q}^{(I) P B 1^{*}}=\operatorname{argmax}\left|\mathcal{A}^{(I)}\right| \\
P B 2: \mathbf{X}_{p q}^{(I) P B 2^{*}}=\operatorname{argmax} A_{p s} \\
P B 3: \mathbf{X}_{p q}^{(I) P B 3^{*}}=\operatorname{argmax} R_{p}^{(I)}
\end{array}\right.
$$

s.t. (C1) (C3).

$P B 1$ is used to maximize the ASU set, $P B 2$ to maximize the remaining available transmission time of each MCU, and $P B 3$ is used to maximize the multicast data rate.

(2) Problems solving

(a) $P B 1$ : To maximize the ASU set, the matching scheme transfers more ISUs to ASUs.

To describe the difficulties of the problem more clearly and conveniently elaborate our solution, we have the following definition:

Definition 5: The ISU that can reach the reward threshold after one-time matching is a feasible ISU (FISU), i.e., $\mathcal{F}^{(I)}=\left\{p \mid p \in \mathcal{V}^{(I)}, h_{p}^{(I)} \geqslant \theta_{p}^{(I)}\right\}$.

In this paper, with finite VRUs, the BS gives a higher preference to ISUs that can more easily reach the reward threshold. An ISU can be an FISU, or may not have been decided by $h_{p}^{(I)}$ whether reach $\theta_{p}^{(I)}$ at the present time slot. The gap between them is denoted by

$$
\delta_{p}^{(I)}=\theta_{p}^{(I)}-h_{p}^{(I)}
$$

Intuitively, the smaller the value of $\delta_{p}^{(I)}$ the easier it is for the ISU to reach $\theta_{p}^{(I)}$. Therefore, the matching order prefers the ISU with a smaller $\delta_{p}^{(I)}$.

Remark 4: When a matching scheme $\mathbf{X}_{p q}^{(I) P B 1^{*}}$ is the theoretical optimum, $\forall p \in \mathcal{V}^{(I)} \Rightarrow \delta_{p}^{(I)}=0$.

(b) $P B 2$ : To maximize the remaining available transmission time of each MCU, i.e., minimize the loss of SS, the BS selects the ISUs with larger $\left|\mathcal{U}_{p}^{(I)}\right|$ for priority match, and VRUs are matched to the ISU with stronger SS.

(c) $P B 3$ : Based on Eq. (2) and Eq. (6), we have

$$
L_{p}^{(i)}=A_{p s} \sum_{s=1}^{\left|\mathcal{C}_{p}\right|} R_{p}=W A_{p s} \sum_{s=1}^{\left|\mathcal{c}_{p}\right|} \log _{2}\left(1+\gamma_{p}\right)
$$

From Eq. (22), we know $L_{p}^{(i)}$ is decided by $A_{p s},\left|\mathcal{C}_{p}\right|, \gamma_{p}$. Because the matching decision is made in a very short time, thus, the change of $A_{p s}$ can be ingnored. However, the change of $\left|\mathcal{C}_{p}\right|$ is obvious, and the change in $\gamma_{p}$ is also caused by the change in $\left|\mathcal{C}_{p}\right|$. Hence, we consider the effect of a change in $\left|\mathcal{C}_{p}\right|$ on $L_{p}^{(i)}$.

Theorem 2: The PDF of the multicast data rate is monotonically decreasing with $\left|\mathcal{C}_{p}\right|$.

Proof: The proof based on the PDF of $\gamma_{p}$. Due to space constraints, we omitted the proof process.

Remark 6: Based on Theorem 2, ISUs with less $\left|\mathcal{C}_{p}\right|$ are preferred. 
(3) Heuristic Search Algorithm: SA-D2MD-M Algorithm

Based on above analysis, to tradeoff the solutions to each of these three problems, we proposed the SA-D2MD user matching algorithm (SA-D2MD-M) to get the solution:

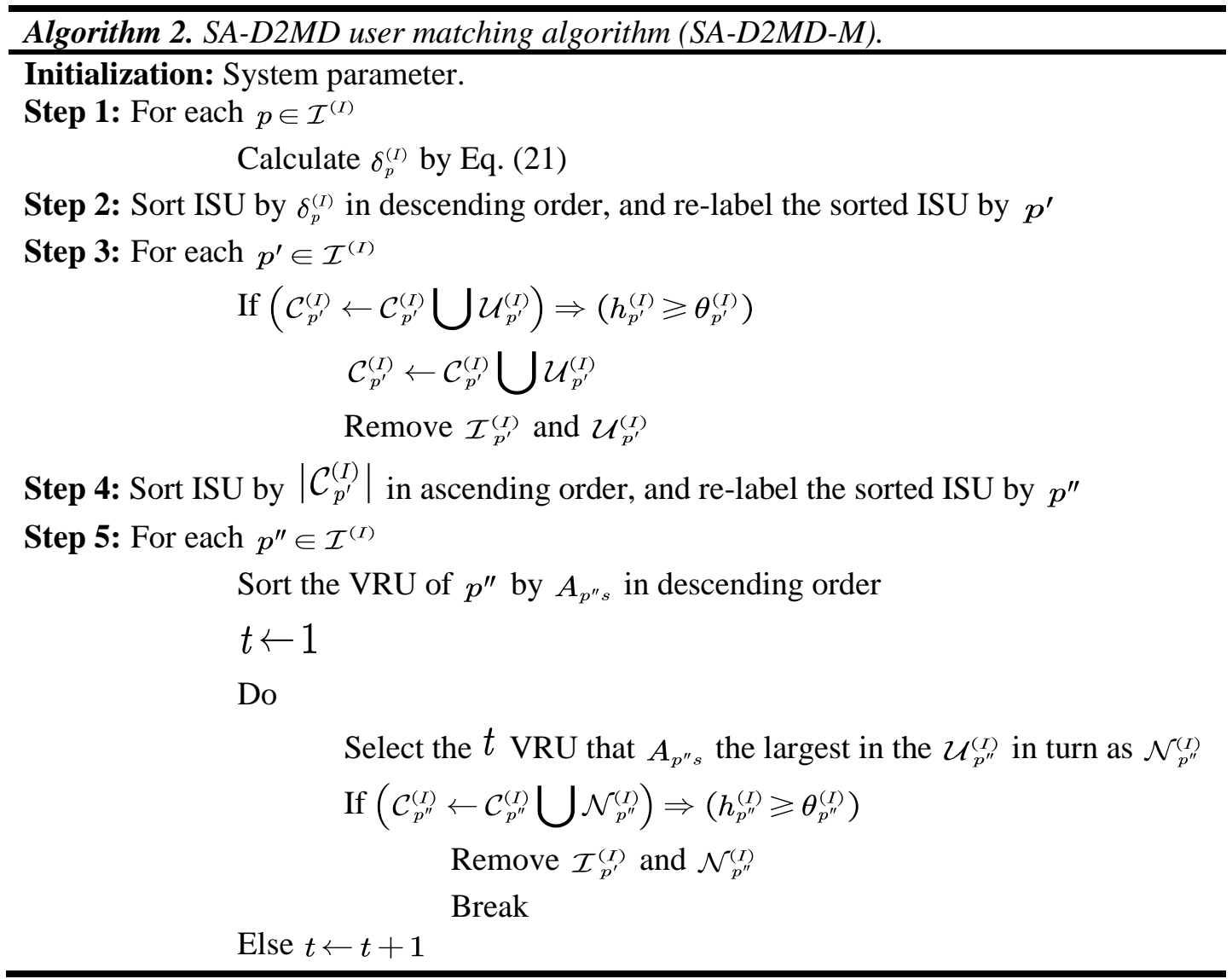

Remark 7: The SA-D2MD-M is an online algorithm. For the step 1, the basic operation is to calculate $\delta_{p}^{(I)}$, and its time complexity can be denoted by $O\left(\left|\mathcal{I}^{(I)}\right|\right)=O(n)$. For the step 3 and step 5, the basic operation is compare, and their time complexity can be denoted by $O\left(\left|\mathcal{I}^{(I)}\right|\right)=O(n)$ respectively. For the step 2 and step 4, the time complexity of general sorting algorithms can be denoted by $O\left(\left|\mathcal{I}^{(I)}\right| \log \left|\mathcal{I}^{(I)}\right|\right)=O(n \log n)$. Therefore, the overall time complexity of the SA-D2MD-M can be denoted by $O(n \log n)$.

\section{Simulations}

In this paper, we built a simulation system based on MATLAB 2017b. The simulation scenario was a single-cell model as shown in Fig. 4. 


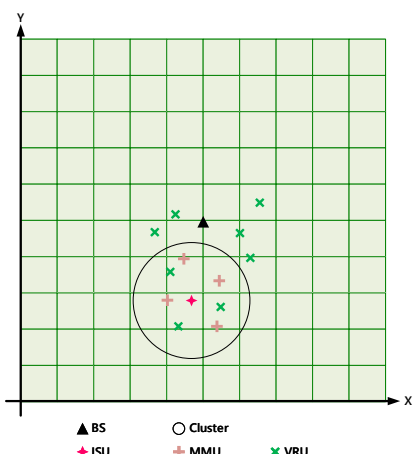

(a) Single-SU

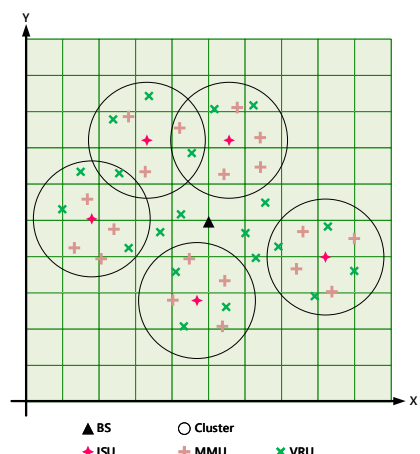

(b) Multi-SU

Fig. 4. SA-D2MD simulation scenario

To test the performance of the proposed algorithms, we divided SA-D2MD simulation scenario into two cases. In Fig. 4(a), it contained single-SU and VRUs, SA-D2MD-S algorithm is used only. In Fig. 4(b), it contained multi-SU and VRUs, SA-D2MD-M\&S algorithms are used. Each ISU stored one video and all VRUs demanded it. The D2D communication mode was selected a priori for all VRUs. Each ISU was matched with some MCUs. The typical values of basic parameters of the simulation are shown in Table 2 and Table 3.

Table 2. Basic parameters [61]

\begin{tabular}{|c|c|}
\hline Parameters & Value \\
\hline \hline$P / N_{0}$ & $10 \mathrm{~dB}$ \\
\hline$\alpha$ & 4 \\
\hline$W$ & $10 \mathrm{MHz}$ \\
\hline
\end{tabular}

Further, the time slot $\tau$ was set to $300 \mathrm{~ms}$. To make the decline of the QoE of the VRUs controllable, $\boldsymbol{T}$ was set to $3000 \mathrm{~ms}$ to guarantee that the mean opinion scores (MOS) of all VRUs were equal or greater than 3.

Table 3. Relationship between MOS and VLD [29]

\begin{tabular}{|c|c|}
\hline MOS & VLD \\
\hline \hline $4 \sim 5$ & $\leqslant 1000$ \\
\hline $3 \sim 4$ & $1000 \sim 3000$ \\
\hline $2 \sim 3$ & $3000 \sim 5000$ \\
\hline $1 \sim 2$ & $5000 \sim 10000$ \\
\hline 1 & $\geqslant 10000$ \\
\hline
\end{tabular}

For the SA-D2MD-S, we found that $\varsigma$ had a strong relation with $\mathbb{E} G_{p s}$ and $\mathbb{D} G_{p s}$. The closed form was difficult to express. Thus, we set $\varsigma=0$ first and then found a numerical solution instead. We plotted the AADT and VLD of the SA-D2MD-S and SA-D2MD-M\&S with different Poisson distribution parameters, expectations, and variances of SS. We plotted the AADT and VLD of the SA-D2MD-M\&S with different numbers of ISUs.

\subsection{Single-SU Scenario}


We compared the SA-D2MD-S algorithm with the following algorithms in terms of AADT and VLD:

(1) Random waiting D2MD algorithm (RW-D2MD): The ISU obverses the VRUs for random time slots and then began the D2MD transmission process.

(2) Non-waiting D2MD algorithm (NW-D2MD): The ISU began the D2MD transmission process immediately after receiving the request of VRUs.

(3) Socially aware probability select algorithm (SA-PS): The ISU selects a subset of VRUs for the D2MD transmission process based on SS. It means if a VRU has the strongest SS with a ISU, the VRU has the biggest probability be grouped to the ISU.

(4) Socially aware peer discovery algorithm (SA-PD) [62]: The ISU obverses VRUs for some time slots based on the SS between ISU and VRUs, and then began the D2MD transmission process. It means if the ISU which has the strongest SS with its associated VRU set, the ISU has the shortest VLD.

\subsubsection{AADT}

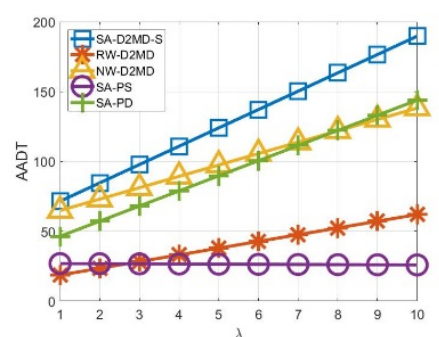

(a)

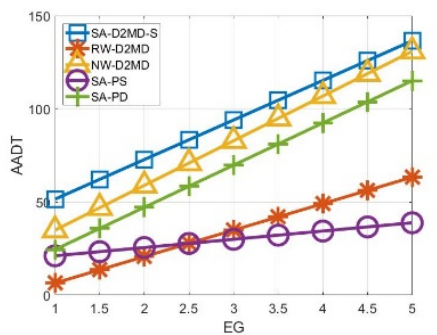

(b)

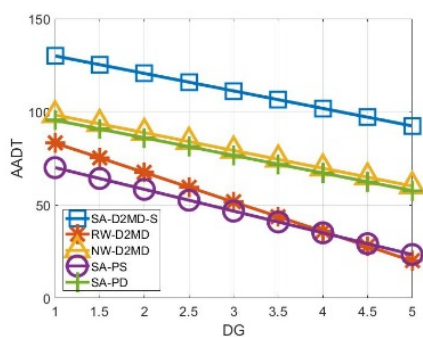

(c)

Fig. 5. The AADT with key parameters increased of SA-D2MD-S

The capacity for offloading BS traffic vary at large with the increase in VRU, the strength and difference of SS. In this paper, we verify the AADT of the proposed scheme. Therefore, in Fig. 5, we analyse the performance of our scheme by varying the number of $\lambda, \mathbb{E} G_{p q}$ and $\mathbb{D} G_{p q}$. Specifically, Fig. 5(a) plots AADT and shows an vary trend with increase in $\lambda$ for all the schemes. It is obvious, as with increase in $\lambda$ the AADT increase. We note that our scheme increase in $\lambda$ leads to higher AADT than NW-D2MD, SA-D2MD and RW-D2MD because our scheme can wait for more VRUs. Moreover, our scheme can wait for VRUs which have stronger SS that leads our scheme higher than SD-PS. In additional, our scheme can avoid the VRU which have stronger SS away, thus, our scheme 24.2\% higher AADT than SA-PD. Fig. 5(b) plots AADT and shows an increasing trend with increase in $\mathbb{E} G_{p q}$ for all the schemes. As $\mathbb{E} G_{p q}$ increase, the AADT of our scheme higher than RW-D2MD and NW-D2MD 53.6\% and $4.1 \%$ respectively. This is mainly due to our scheme play the role of SS, the VRUs which have strong SS can be waited for. But, it is interesting to note that, when $\mathbb{E} G_{p q}$ gets big enough, the AADT of our scheme and NW-D2MD are much the same. That is because our scheme incurred the more VRUs are grouped, the higher probability of poor SNR is accessed. Therefore, the effect of our scheme on offloading BS traffic is reduced. Moreover, our scheme better than SA-PS and SA-PD that is because our scheme can wait for VRUs with better SNR, i.e., they don't make the multicast SNR get worse. From Fig. 5(c), it is evident that, the AADT of all scheme get worse with $\mathbb{D} G_{p q}$ increased. This can be explained as, with the increase in 
$\mathbb{D} G_{p q}$, the difference of SS gets bigger. This makes the time they leave the ASU very different, this means multicast advantage will decline as the number of VRU decreases until it degenerates into unicast. In spite of this, because our scheme can group VRUs which have higher SNR with the ASU, thus, our scheme has the best AADT of all. As shown in the Fig. 5(c), our scheme is $78.4 \%, 34.9 \%, 74.7 \%$, and $37.6 \%$ higher than the others, respectively.

\subsubsection{VLD}

VLD is the cost of optimization of this paper. As shown in Fig. 6, the VLD of SA-D2MD-S exceeded those of all other algorithms except RA-D2MD.

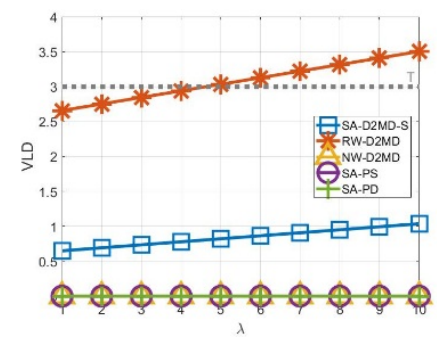

(a)

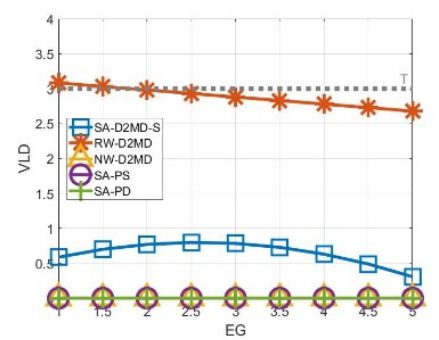

(b)

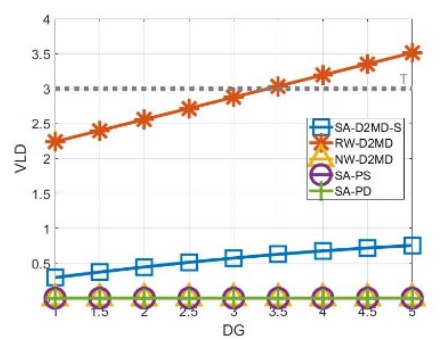

(c)

Fig. 6. The VLD with key parameters increased of SA-D2MD-S

In Fig. 6, we analyse the cost of our scheme by varying the number of $\lambda, \mathbb{E} G_{p q}$ and $\mathbb{D} G_{p q}$. Specifically, Fig. 6(a) depicts the increasing trend of VLD as increase in $\lambda$. That is because the more VRUs will have higher probability to incur worse SNR, thus, the ISU will cost more time to find better VRUs to reach the reward threshold. Fig. 6(b) plots the relation between VLD and $\mathbb{E} G_{p q}$. For the proposed scheme, the VLD increases slowly with the increase in $\mathbb{E} G_{p q}$ due to the fact that, if a VRU has poor SNR and high SS with the ISU, instead of increasing rewards, it reduces them. Then as $\mathbb{E} G_{p q}$ continues to increase, the benefits ISU gets from SS outweigh the aforementioned costs, hence, the VLD decrease. Fig. 6(c) plots the relation between VLD and $\mathbb{D} G_{p q}$. It is evident that, the VLD increase gradually. This can be explained as, because the end time of a D2MD process depends on the last VRU to leave, hence, with the increase in $\mathbb{D} G_{p q}$, more VRUs are leaving too soon. This makes ASU multicast to a very small number of VRUs for a longer period of time, even unicast. ISUs try to avoid the above situation, so it costs longer VLD to find VRUs which have higher SNR and stronger SS.

\subsection{Multi-SU Scenario}

We compared the SA-D2MD-M\&S algorithm with following algorithms in terms of AADT and VLD after user grouping.

(1) Random D2MD algorithm (RW-D2MD) + NW-D2MD: The VRUs are matched to ISUs randomly, which then began the D2MD transmission process.

(2) Physical distance-based D2MD algorithm (PD-D2MD) + NW-D2MD: The VRUs are matched to ISUs based on physical distance, which then began the D2MD transmission process.

(3) Social distance-based D2MD algorithm (SD-D2MD) + NW-D2MD [47]: The VRUs are matched to ISUs based on SS, and then began the D2MD transmission process. 
(4) Physical social-distance-based-CRP D2MD algorithm (PSD-CRP-D2MD) + NWD2MD [46]: The VRUs are matched to ISUs based on SS, which is the matching probability. The ISUs the began the D2MD transmission process.

(5) PD-D2MD + SA-D2MD-S: The VRUs were matched to the ISUs based on PD-D2MD. The ISUs then began the D2MD transmission processes based on the SA-D2MD-S.

\subsubsection{AADT}

The maximization of AADT was the object of optimization in this paper. As shown in Fig. 7, the AADT using SA-D2MD-M\&S nearly always exceeded those of other algorithms.

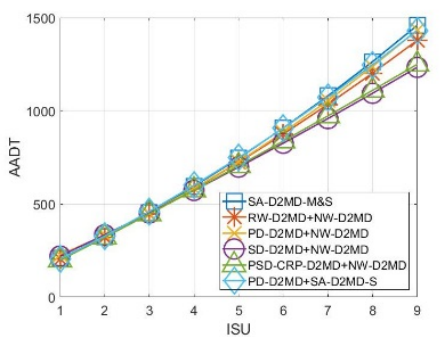

(a)

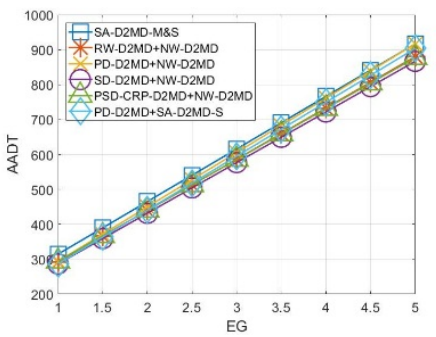

(c)

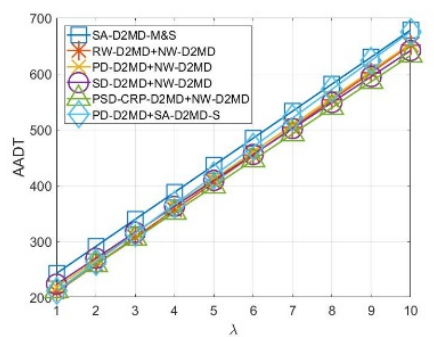

(b)

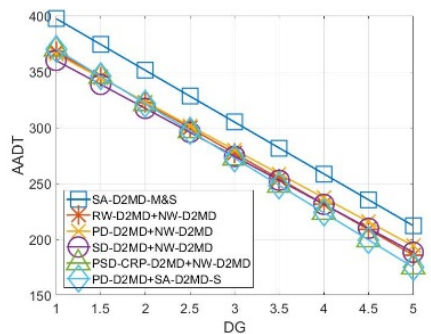

(d)

Fig. 7. The AADT with key parameters increased of SA-D2MD-M\&S

We further analyse the AADT of our scheme by varying the number of $\left|\mathcal{I}^{(I)}\right|, \lambda, \mathbb{E} G_{p q}$ and $\mathbb{D} G_{p q}$. The capacity for offloading BS traffic also vary at large with the increase in SU. As $\left|\mathcal{I}^{(I)}\right|$ increased, when $\left|\mathcal{I}^{(I)}\right| \geqslant 4$, the AADT using SA-D2MD-M\&S exceeded all algorithms. This is because there are more ISUs are provided to the VRUs, and SA-D2MD-M can match better access options for the VRUs from a spatial perspective. As shown in Fig. 7(a), when $\left|\mathcal{I}^{(I)}\right|=9, \mathbb{E} G_{p q}=2, \mathbb{D} G_{p q}=2, \lambda=3$, the AADT using SA-D2MD-M\&S was higher than other algorithms by $5.5 \%, 1.7 \%, 15.4 \%, 14.3 \%$, and $1.9 \%$ respectively. Changes in $\lambda, \mathbb{E} G_{p q}$ and $\mathbb{D} G_{p q}$ are similar to those in Section 6.1.1 and will not be repeated here.

\subsubsection{VLD}

The VLD was the cost of optimization of this paper. As shown in Fig. 8, the VLD of SAD2MD-M\&S exceeded those of the other algorithms except for PD-D2MD + SA-D2MD-S. 


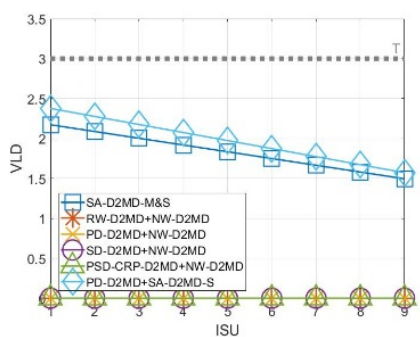

(a)

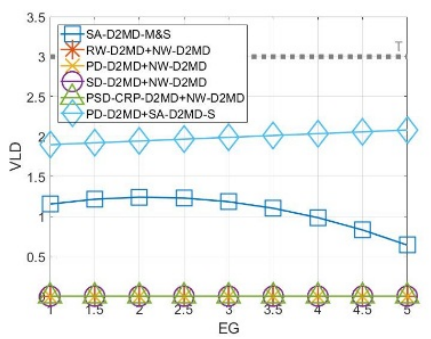

(c)

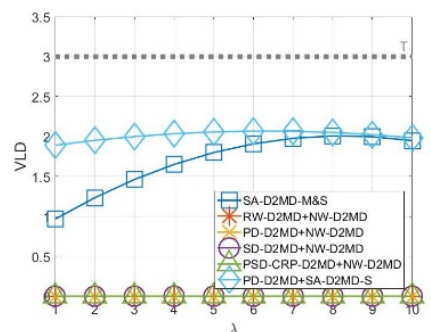

(b)

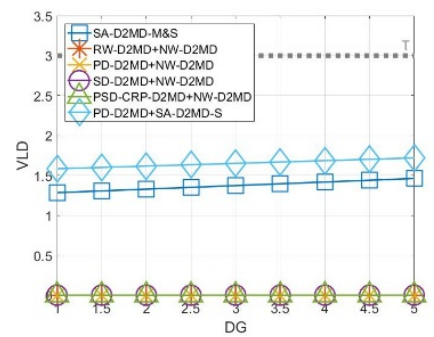

(d)

Fig. 8. The VLD with key parameters increased of SA-D2MD-M\&S

We further analyse the VLD of our scheme by varying the number of $\left|\mathcal{I}^{(I)}\right|, \lambda, \mathbb{E} G_{p q}$ and $\mathbb{D} G_{p q}$. It also vary at large with the increase in SU. As $\left|\mathcal{I}^{(I)}\right|$ increased, the VLD using SAD2MD-M\&S gradual decline. We note that our scheme increase in $\lambda$ leads to lower VLD than PD-D2MD + SA-D2MD-S. This is because SA-D2MD-M match order prefers the ISU with a smaller $\delta_{p}^{(I)}$, and make the ISU transfer to ASU faster. As shown in Fig. 8(a), when $\left|\mathcal{I}^{(I)}\right|=9, \mathbb{E} G_{p q}=2, \mathbb{D} G_{p q}=2, \lambda=3$, the VLD using SA-D2MD-M\&S was lower than PD-D2MD + SA-D2MD-S by $6.3 \%$. Changes in $\lambda, \mathbb{E} G_{p q}$ and $\mathbb{D} G_{p q}$ are similar to those in Section 6.1.2 and will not be repeated here.

\section{Conclusion}

A method for offloading BS traffic while distributing popular videos and guaranteeing the QoE for asynchronous user requests was proposed in this paper, we designed a user grouping scheme to match ISUs and VRUs in BS-assisted D2MD by exploiting SS and the VLD-tolerant property of VRUs. We formulated a time-varying bipartite graph-matching problem to maximizing the AADT while guaranteeing the QoE of the VRUs. Based on the system model, the problem was decoupled into two subproblems. For the first subproblem, we proposed the SA-D2MD-S algorithm to obtain a higher AADT. Finite horizon optimal stopping theory was used to select the MMU set for the ISU. For the second subproblem, an optimization problem was proposed to maximize the AADT of the ISUs. A heuristic SA-D2MD-M algorithm was proposed to solve it. The results of simulations showed that compared with other user matching algorithms, the SA-D2MD-S and SA-D2MD-M\&S algorithms exhibited advantages in terms of AADT while guaranteeing low VLD.

However, because we assume the long-term VRU arriving and SS between VRU and SU are stable. It is not fit for sudden changes in VRU arriving and SS such as emergency 
communications. Hence, in future work, for network optimization in real time, other methods will be introduced, such as reinforcement learning. To further offload BS traffic, social altruism will be considered, and an incentive mechanism will be studied for SU selection and the update of D2MD video distribution. To further extend the application scenarios of this scheme, more business features can be considered, such as multiple videos and varying popularity.

\section{References}

[1] Yonggang Wen, Xiaoqing Zhu, and Joel J. P. C. Rodrigues, “Cloud Mobile Media: Reflections and Outlook,” IEEE Transactions on Multimedia, vol.16, no.4, pp.885-902, 2014.

[2] Liqiang Nie, Meng Liu, and Xuemeng Song, "Multimodal Learning toward Micro-Video Understanding," MORGAN \& CLAYPOOL PUBLISHERS, 2019.

[3] Cisco Visual Networking Index: Global Mobile Data Traffic Forecast Update, 2017-2022, Cisco Syst., San Jose, USA, Feb. 2019.

[4] M. Cha, H. Kwak, P. Rodriguez, Y. Ahn, and S. Moon, "I tube, you tube, everybody tubes: Analyzing the world's largest user generated content video system," in Proc. of 7th ACM SIGCOMM Conf. Internet Meas., pp. 1-14, 2007.

[5] Kurdoglu. E, Yong Liu, and Yao Wang, "Dealing with User Heterogeneity in P2P Multi-Party Video Conferencing: Layered Distribution Versus Partitioned Simulcast," IEEE Transactions on Multimedia, vol.18, no.1, pp.90-101, Jan. 2016. Article (CrossRef Link)

[6] Koichiro Kitagawa, Hiroaki Homma, Yasuhiro Suegara, and Yoji Kishi, "A User Selection Algorithm for D2D Multicast Communication Underlaying Cellular Systems," in Proc. of 2017 IEEE Wireless Communications and Networking Conference (WCNC), pp.1-6, Mar. 2017. Article (CrossRef Link)

[7] Sara Pizzi, Federica Rinaldi, Antonella Molinaro, Antonio Iera, and Giuseppe Araniti, "EnergyEfficient Multicast Service Delivery Exploiting Single Frequency Device-To-Device Communications in 5G New Radio Systems," Sensors, vol.18, no.2205, pp.1-15, Jul. 2018.

Article (CrossRef Link)

[8] Mehdi Naderi Soorki, Mohammad Hossein Manshaei, Walid Saad, Hossein Saidi, Ramin Hasibi, Amirhosein Shafieyoun, and Amirreza Hajrasouliha, "Collaborative Real-Time Content Download Application for Wireless Device-to-Device Communications," in Proc. of GLOBECOM 20172017 IEEE Global Communications Conference, pp.1-6, Dec. 2017. Article (CrossRef Link)

[9] Filippo Rebecchi, Marcelo Dias de Amorim, and Vania Conan, "Should I seed or should I not: On the remuneration of seeders in D2D offloading," in Proc. of 2016 IEEE 17th International Symposium on A World of Wireless, Mobile and Multimedia Networks (WoWMoM), pp.1-9, Jul. 2016. Article (CrossRef Link)

[10] Yanyao Shen, Chunxiao Jiang, Tony Q. S. Quek, and Yong Ren, "Device-to-Device-Assisted Communications in Cellular Networks: An Energy Efficient Approach in Downlink Video Sharing Scenario," IEEE Transactions on Wireless Communications, vol.15, no.2, pp.1575-1587, Feb. 2016. Article (CrossRef Link)

[11] Yanli Xu, and Feng Liu, "QoS Provisionings for Device-to-Device Content Delivery in Cellular Networks,” IEEE Transactions on Multimedia, Vol.19, no.11, pp.2597-2608, Nov. 2017. Article (CrossRef Link)

[12] Kuan Wu, and Ming Jiang, "Joint Resource Efficiency Optimisation for Overlay Device-to-Device Retransmissions," in Proc. of 2017 IEEE 85th Vehicular Technology Conference (VTC Spring), pp.1-5, Jun. 2017. Article (CrossRef Link)

[13] Wen Ji, Bo-Wei Chen, Xiangdong Wang, Haiyong Luo, Mucheol Kim, and Yiqiang Chen, "CrossLayer Opportunistic Scheduling for Device-to-Device Video Multicast Services," ACM Transactions on Embedded Computing Systems, vol.15, no.2, pp.37, May. 2016.

Article (CrossRef Link) 
[14] Claudio Ettore Casetti, Carla Fabiana Chiasserini, Yufeng Duan, Paolo Giaccone, and Andres Perez Manriquez, "Data Connectivity and Smart Group Formation in Wi-Fi Direct Multi-Group Networks,” IEEE Transactions on Network and Service Management, vol.15, no.1, pp.245-259, 2017. Article (CrossRef Link)

[15] Zicheng Xia, Jiawei Yan, and Yuan Liu, "Cooperative Content Delivery in Multicast Multihop Device-to-Device Networks,” IEEE Access, vol.5, pp.6314-6324, Feb. 2017. Article (CrossRef Link)

[16] Sanaa Sharafeddine, and Omar Farhat, "A proactive scalable approach for reliable cluster formation in wireless networks with D2D offloading,” Ad Hoc Networks, vol.77, pp.42-53, Aug. 2018. Article (CrossRef Link)

[17] Salam Doumiati, Hassan Artail, and Karim Kabalan, “A framework for clustering LTE devices for implementing group D2D communication and multicast capability," in Proc. of 2017 8th International Conference on Information and Communication Systems (ICICS), pp.216-221, Apr. 2017.

[18] Shuai Yu, Rami Langar, and Xin Wang, “A D2D-Multicast Based Computation Offloading Framework for Interactive Applications," in Proc. of 2016 IEEE Global Communications Conference (GLOBECOM), pp.1-6, Dec. 2016. Article (CrossRef Link)

[19] Ajay Bhardwaj, and Samar Agnihotri, "Interference-Aware D2D Multicast Session Provisioning in LTE-A Networks," in Proc. of 2017 IEEE Wireless Communications and Networking Conference (WCNC), pp.1-6, Mar. 2017. Article (CrossRef Link)

[20] Ajay Bhardwaj, Student Member, and Samar Agnihotri, "Energy- and Spectral-Efficiency TradeOff for D2D-Multicasts in Underlay Cellular Networks,” IEEE Wireless Communications Letters, vol.7, no.4, pp.546- 549, Aug. 2018. Article (CrossRef Link)

[21] Ajay Bhardwaj, and Samar Agnihotri, "A resource allocation scheme for multiple device-to-device multicasts in cellular networks,” in Proc. of 2016 IEEE Wireless Communications and Networking Conference, pp.1-6, Apr. 2016. Article (CrossRef Link)

[22] Jong-ho Kim, Jingon Joung, and Jeong Woo Lee, "Resource Allocation for Multiple Device-toDevice Cluster Multicast Communications Underlay Cellular Networks,” IEEE Communications Letters, vol.22, iss.2. pp.412-415, Feb. 2018. Article (CrossRef Link)

[23] Hao Feng, Zhiyong Chen, and Hui Liu, "Design and Optimization of VoD Schemes with Client Caching in Wireless Multicast Networks,” IEEE Transactions on Vehicular Technology, vol.67, no.1, pp.765-780, Jan. 2018. Article (CrossRef Link)

[24] Jagadeesha R. Bhat, Jang-Ping Sheu, and Wing-Kai Hon, "Resource Allocation Schemes for Revenue Maximization in Multicast D2D Networks,” IEEE Access, vol.5, pp.26340-26353, Nov. 2017. Article (CrossRef Link)

[25] Chenglin Li, Hongkai Xiong, Junni Zou, and Dapeng Oliver Wu, "Joint Dynamic Rate Control and Transmission Scheduling for Scalable Video Multirate Multicast Over Wireless Networks," IEEE Transactions on Multimedia, vol.20, no.2, pp.361-378, Feb. 2018. Article (CrossRef Link)

[26] Pan Zhao, Lei Feng, Peng Yu, Wenjing Li, and Xuesong Qiu, "Resource allocation for energyefficient device-to-device multicast communication,” in Proc. of 2016 19th International Symposium on Wireless Personal Multimedia Communications (WPMC), pp.1-6, Nov. 2016.

[27] Monia Hamdi, Di Yuan, and Mourad Zaied, "GA-based scheme for fair joint channel allocation and power control for underlaying D2D multicast communications," in Proc. of 2017 13th International Wireless Communications and Mobile Computing Conference (IWCMC), pp.446451, Jun. 2017. Article (CrossRef Link)

[28] Yong Li, Ting Wu, Pan Hui, Depeng Jin, and Sheng Chen, “A Survey on Socially Aware Deviceto-Device Communications,” IEEE Communications Surveys \& Turorials, vol.20, no.3, pp.21692197, 2018. Article (CrossRef Link)

[29] Fan Li, Fu Shuang, Ziyi Liu, and Xueming Qian, “A Cost-Constrained Video Quality Satisfaction Study on Mobile Devices,” IEEE Transactions on Multimedia, pp.1154-1168, vol.20, no.5, May. 2018. Article (CrossRef Link)

[30] China Video Service User's Experience Standards Working Group, China Video Service Experience, Post \& Telecom Press, China, Apr. 2017. 
[31] Zaijian Wang, Shiwen Mao, Lingyun Yang, and Pingping Tang, "A survey of multimedia big data," China Communications, vol.15, no.1, pp.155-176, Jan. 2018. Article (CrossRef Link)

[32] Zhi Wang, Jiangchuan Liu, and Wenwu Zhu, "Social-aware video delivery: challenges, approaches, and directions," IEEE Network, vol.30, no.5, pp.35-39, Sep-Oct. 2016. Article (CrossRef Link)

[33] Jun Li, Miao Liu, Jinhui Lu, Feng Shu, Yijin Zhang, Siavash Bayat, and Dushantha Nalin K. Jayakody, "On Social-Aware Content Caching for D2D-Enabled Cellular Networks with Matching Theory,” IEEE Internet of Things Journal, vol.6, no.1, pp.297-310, Feb. 2019.

Article (CrossRef Link)

[34] Wenrong Gong, Guomin Li, and Baiping Li, "System utility based resource allocation for D2D multicast communication in softwaredefined cellular networks," AEU - International Journal of Electronics and Communications, vol.96, pp.138-143, Aug. 2018. Article (CrossRef Link)

[35] Yiming Zhao, and Wei Song, "Social-Aware Energy-Efficient Data Dissemination with D2D Communications," in Proc. of 2016 IEEE 83rd Vehicular Technology Conference (VTC Spring), pp.1-5, 2016. Article (CrossRef Link)

[36] Muluneh M. Tulu, Ronghui Hou, Chuanyin Li, and Melkamu D. Amentie, "Cluster head selection method for content-centric mobile social network in 5G," IET Communications, vol.12, no.4, pp.402-408, Feb. 2018. Article (CrossRef Link)

[37] Hongcheng Huang, Biao Liu, Min Hu, Yang Tao, and Wei Xiang, "User-Information-Aware D2D Multicast File Distribution Mechanism,” Sensors, vol.18(10), pp.1-20, Oct. 2018.

Article (CrossRef Link)

[38] Lei Feng, Pan Zhao, Fanqin Zhou, Mengjun Yin, Peng Yu, Wenjing Li, and Xuesong Qiu, "Resource Allocation for 5G D2D Multicast Content Sharing in Social-Aware Cellular Networks," IEEE Communications Magazine, vol.56, no.3, pp.112-118, Mar 2018. Article (CrossRef Link)

[39] Yan Wu, Dan Wu, Lianxin Yang, Xin Shi, Liang Ao, and Qinxue Fu, "Matching-Coalition Based Cluster Formation for D2D Multicast Content Sharing,” IEEE Access, vol.7, pp.73913-73928, Jun. 2019. Article (CrossRef Link)

[40] Bo Bai, Li Wang, Zhu Han, Wei Chen, and Tommy Svensson, "Caching based socially-aware D2D communications in wireless content delivery networks: a hypergraph framework," IEEE Wireless Communications, vol.23, no.4, pp.74-81, Aug. 2016. Article (CrossRef Link)

[41] Lianxin Yang, Dan Wu, Shiming Xu, Guangchun Zhang, and Yueming Cai, "Social-EnergyAware User Clustering for Content Sharing Based on D2D Multicast Communications," IEEE Access, vol.6, pp.36092-36104, Jul. 2018. Article (CrossRef Link)

[42] Yang Cao, Tao Jiang, Xu Chen, and Junshan Zhang, "Social-Aware Video Multicast Based on Device-to-Device Communications," IEEE Transactions on Mobile Computing, vol.15, no.6, pp.1528-1539, Jun. 2016. Article (CrossRef Link)

[43] Lindong Zhao, LeiWang, Xuguang Zhang, and Bin Kang, "Social-Aware Cooperative Video Distribution via SVC Streaming Multicast," Wireless Communications and Mobile Computing, vol.2018, pp.1-9, Oct. 2018. Article (CrossRef Link)

[44] Jingjing Wang, Yanjing Sun, Bowen Wang, Bin Wang, Anyi Wang, Song Li, and Zhi Sun, "Resource Allocation for D2D Video Multicast Using Multi-Leader Multi-Follower Stackelberg Game with Personalized Incentives,” IEEE Access, vol.7, pp.117019-117028, Sep. 2019. Article (CrossRef Link)

[45] Giuseppe Araniti, Antonino Orsino, Leonardo Militano, Li Wang, and Antonio Iera, "ContextAware Information Diffusion for Alerting Messages in 5G Mobile Social Networks," IEEE Internet of Things Journal, vol.4, no.2, pp.427-436, Apr. 2017. Article (CrossRef Link)

[46] Fan Jiang, Yao Liu, Chenbi Li, and Changyin Sun, "Energy-Efficient Multicast Transmission for Underlay Device-to-Device Communications: A Social-Aware Perspective,” Mobile Information Systems, vol.2017, pp.1-17, Mar. 2017. Article (CrossRef Link)

[47] Li Wang, Giuseppe Araniti, Chunyan Cao, Wei Wang, and Yang Liu, "Device-to-Device Users Clustering Based on Physical and Social Characteristics," International Journal of Distributed Sensor Networks, vol.11, no.8, pp.1-14, May. 2015. Article (CrossRef Link)

[48] D. M. Blei and P. I. Frazier, "Distance dependent Chinese restaurant processes," Journal of Machine Learning Research, vol.12, pp. 2461- 2488, 2012. Article (CrossRef Link) 
[49] Fuxin Ren, Xiaoxiang Wang, Dongyu Wang, Yufang Zhang, and Yanwen Lan, “Joint Social, Energy and Transfer Rate to Select Cluster Heads in D2D Multicast Communication,” in Proc. of 2018 10th International Conference on Measuring Technology and Mechatronics Automation, pp.434-439, Feb. 2018. Article (CrossRef Link)

[50] Yanru Zhang, Erte Pan, Lingyang Song, Walid Saad, Zaher Dawy, and Zhu Han, "Social Network Aware Device-to-Device Communication in Wireless Networks," IEEE Transactions on Wireless Communications, vol.14, no.1, pp.177-190, Jan. 2015. Article (CrossRef Link)

[51] Zufan Zhang, and Lisha Wang, "Social tie-driven content priority scheme for D2D communications,” Information Sciences, vol.480, pp.160-173, Apr. 2019. Article (CrossRef Link)

[52] Yiming Zhao, Wei Song, and Zhu Han, "Social-Aware Data Dissemination via Device-to-Device Communications: Fusing Social and Mobile Networks with Incentive Constraints," IEEE Transactions on Services Computing, vol.12, no.3, pp.489-502, 2019. Article (CrossRef Link)

[53] Dapeng Wu, Junjie Yan, Honggang Wang, Dalei Wu, and Ruyan Wang, "Social Attribute Aware Incentive Mechanism for Device-to-Device Video Distribution," IEEE Transactions on Multimedia, vol.19, no.8, pp.1908-1920, Aug. 2017. Article (CrossRef Link)

[54] Yichao Chen, Shibo He, Fen Hou, Zhiguo Shi, and Jiming Chen, "Promoting Device-to-Device Communication in Cellular Networks by Contract-based Incentive Mechanisms,” IEEE Network, vol.31, no.3, pp.14-20, May/Jun. 2017. Article (CrossRef Link)

[55] Konglin Zhu, Wenting Zhi, Xu Chen, and Lin Zhang, "Socially Motivated Data Caching in UltraDense Small Cell Networks,” IEEE Network, vol.31, no.4, pp.42-48, Jul/Aug. 2017. Article (CrossRef Link)

[56] Omid Semiari, Walid Saad, Stefan Valentin, Mehdi Bennis, and H. Vincent Poor, "Context-Aware Small Cell Networks: How Social Metrics Improve Wireless Resource Allocation,” IEEE Transactions on Wireless Communications, vol.14, no.11, pp.5927-5940, Nov. 2015. Article (CrossRef Link)

[57] Mengyuan Zhang, Xu Chen, and Junshan Zhang, "Social-aware relay selection for cooperative networking: An optimal stopping approach,” in Proc. of 2014 IEEE International Conference on Communications (ICC), pp.2257- 2262, Jun. 2014. Article (CrossRef Link)

[58] T. S. Ferguson. Optimal stopping and applications, Mar. 12, 2014. [Online]. Available: http://www.math.ucla.edu/tom/Stopping/Contents.html

[59] P. Walters. An Introduction to Ergodic Theory. Springer, Jun. 2003.

[60] Sheldon Ross, Introduction to Probability Models, the 11th edition, Academic Press, Jan. 2014.

[61] He Zhang, Qinghe Du, Pinyi Ren, and Zehua Wang, "Social-Aware D2D Relay Networks for Stability Enhancement: An Optimal Stopping Approach,” IEEE Transactions on Vehicular Technology, vol.67, no.9, pp.8860-8874, Sep. 2018.

[62] Bentao Zhang, Yong Li, Depeng Jin, Pan Hui, and Zhu Han, "Social-Aware Peer Discovery for D2D Communications Underlaying Cellular Networks,” IEEE Transactions on Wireless Communications, vol.14, no.5, pp.2426-2439, May. 2015. Article (CrossRef Link) 


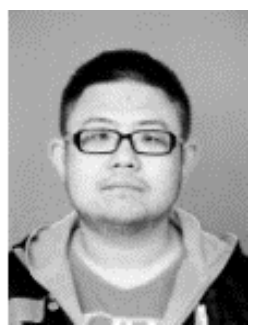

Jianlong Liu received the B.S. degree in computer science and technology from Beijing Information Science and Technology University, Beijing, China and the M.S. degree in software engineering from Inner Mongolia University, Hohhot, Inner Mongolia, China. He is currently pursuing the Ph.D. degree in computer science and technology at Beijing University of Posts and Telecommunications. His research interests include wireless resource management in 5G, D2D communication, mobile content distribution network and social computing.

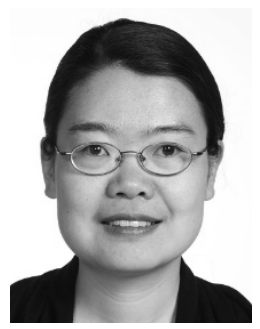

Wen'an Zhou is currently an associate Professor and the director of ICN\&SEC center of School of Computer Science, BUPT. She received her Ph.D. degree in electrical engineering from Beijing University of Posts and Telecommunications (BUPT) in 2002, M.S. degree in telecommunication and electronic system from CATT in 1997, and Bachelor degree in electronic automatics and Bachelor degree in economic management from Tianjin University in 1994. Before joining the faculty at BUPT in 2002, she had worked for China Academy of Telecommunication Technologies (CATT) in R\&D on IS-95 cellular system for 3 years (from 1997 to 1999). In 2007, she furthered her study of broadband wireless communication technology in the University of California, San Diego (UCSD) as a Visiting Scholar, which is sponsored by the national Young Teacher Program. Her current research interests include wireless mobile communication theory, Radio Resource Management and QoE management in $5 \mathrm{G}$, etc.

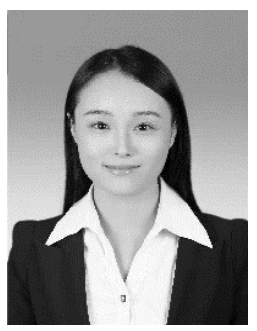

Lixia Lin was born in Dezhou, China, in 1989. She received the B.S. degree in automation from Qingdao University of Technology, Qingdao, China, and the M.S. degree in electronic and communication engineering from Beijing Electronics Science \& technology Institute, Beijing. She is currently pursuing the Ph.D. degree in computer science and technology with the Beijing University of Posts and Telecommunications.

Her research interests include network resource allocation and scheduling in PONs and wireless. 